\title{
Picture-word differences in a sentence verification task
}

\author{
PAULA GOOLKASIAN \\ University of North Carolina, Charlotte, North Carolina
}

\begin{abstract}
Effects of picture-word format were investigated with four problem-solving items. In Experiment 1, picture-word input was presented for $8 \mathrm{sec}$ followed by a test sentence that included verbatim and inference statements. Subjects made a true/false reaction time to the test sentence. In Experiment 2, the input remained on the screen while the test sentence was presented with varied stimulus onset asynchronies from 0 to $1,000 \mathrm{msec}$. Results showed that responses to pictures were faster than responses to words, and the format effect was larger with inference than with verbatim sentences. The picture advantage seemed to be due to the nature of the input and how information is extracted from it. The findings are discussed within the context of text-processing theories (Glenberg \& Langston, 1992; Larkin \& Simon, 1987).
\end{abstract}

Although format effects have been identified in previous research (Goolkasian \& Park, 1980; Kroll \& Corrigan, 1981; Pellegrino, Rosinski, Chiesi, \& Siegel, 1977; Potter \& Falconer, 1975; Smith \& Magee, 1980), the results have varied with the kind of task, and there is a lingering debate regarding the nature of the representations that are developed from picture and word versions of stimuli. Dual-coding theory (Paivio, 1971, 1975, 1978) proposed separate but interconnected verbal and symbolic systems in which pictures have some processing advantage because they generate representations in both memory systems, whereas words have only verbal representations. An alternative multimodal theory (Potter, 1979; Seymour, 1973; Snodgrass, 1984) proposed that, in addition to the verbal and symbolic representation, there is a third propositional memory system in which concepts are amodal and equally accessible from both words and pictures. Hypothesizing from a current version of this theory, Theios and Amrhein (1989b) have suggested that format differences occur when naming responses are required, because picture naming involves two additional processes not present in naming words: determining the meaning of the picture, and finding a name for the picture. Whenever the task requires conceptual processing, format effects disappear because of an amodal abstract conceptual processor. These experiments extend the previous work with picture-word processing by testing whether format effects occur with problem-solving items that require some memory for background inputs but also require reasoning.

This work was supported in part by funds from the Foundation of The University of North Carolina at Charlotte and from the State of North Carolina. Some of these findings were reported at the annual meeting of the Psychonomic Society in St. Louis, November 1994. The author thanks Holly Green and Helen Summer Eubanks for their assistance with this research. Correspondence should be sent to P. Goolkasian, Department of Psychology, UNCC, 9201 University City Boulevard, Charlotte, NC 28223-0001 (e-mail: fpy00pag@unccvm.uncc.edu).
Clark and Chase (1972) were the first to use the sentence-picture verification task to study conceptual codes generated by pictures and words. In their task, subjects decided if a test sentence was true or false with respect to a picture. Their results suggested that subjects' decisions were made through a series of discrete stages in which both sentence and picture are encoded into a common abstract representation. The model of sentence-picture comparison (Carpenter \& Just, 1975) developed from their data is consistent with the multimodal theory of picture-word processing.

In this study, the sentence-picture verification task was modified so that it incorporated more varied examples of problem solving than the one concept (above and below) used by Clark and Chase (1972), and it included a direct test of format effects by requiring subjects to compare a test sentence to either a picture or a word input rather than the picture input tested by Clark and Chase. Also, the test statements were all positive, whereas Clark and Chase included negative statements. The purpose of this study was to test whether format effects would be obtained when problem-solving items required reasoning from pictureword inputs. Format effects would not be predicted by multimodal theories such as Theios and Amrhein (1989b) because access to the semantic network would be abstract and amodal, whereas other theories (Glaser \& Glaser, 1989; Paivio, 1971, 1975, 1978) predict that pictures would access the semantic network more readily than words. Glaser and Glaser (1989) have developed a theory of picture-word processing in which two separate but interconnected memories are proposed and words are believed to have privileged access to the lexicon, whereas pictures and colors have privilege access to the semantic network. Results of the previous studies of picture-word processing may be specific to the kind of stimuli and tasks that were studied. Theios and Amrhein (1989b) used simple shapes, whereas Smith and Magee (1980) used names of animals and articles of clothing, and Glaser and Glaser 
(1989) used Stroop stimuli with separated target and distractor elements. Although these stimuli might be useful for studying simple naming effects and basic categorizing judgments, they do not require complex reasoning that might tell us about format effects that occur when students are presented with material in either picture or word format and are required to problem solve or reason with these materials. The rationale for this study came from a consideration of recent theories of picture-word processing (Glaser \& Glaser, 1989; Theios \& Amrhein, $1989 \mathrm{~b}$ ) together with studies of text processing that have shown a consistent advantage in comprehension when pictures are present in the text.

Studies of text comprehension have shown that pictures facilitate comprehension. Glenberg and Langston (1992) developed a mental model to describe how pictures aid understanding and retention of textual material. Although the representations are propositional, when pictures are integrated into the text, representations of the material are richer and more elaborate than are representations of text presented alone. Another theory (Larkin \& Simon, 1987) explains the picture advantage in the way information is extracted from pictures and words. Text and diagrams containing the same information are not necessarily equivalent in terms of the processing required to extract the information. For example, some features may be directly represented in one that may be inferred in the other. They identify picture-word differences in the efficiency of the search for information and differences in the explicitness of the information.

In this study, stimulus materials were developed from problem-solving items used in fuzzy trace theory. There is some evidence for format effects with these materials. Brainerd and Reyna (1993), in working with children, suggested that when information is presented pictorially, memory improves but reasoning is impaired. Memory and reasoning were tested independently through the use of verbatim and gist representations of inputs. According to fuzzy trace theory, gist and verbatim statements are independent and quite different. Verbatim traces are considered to be exogenous because they arise from information that has just been encoded, whereas gist is endogenous because it deals with patterns that the subject retrieves. Problem solving involves processing gists that develop with the encoding of background inputs; however, the gists are not necessarily associated with verbatim traces of those inputs (Brainerd \& Reyna, 1992). This study used both verbatim and inference items.

Four problem-solving items were used: probability judgments with colors and with shapes, category inclusion, and pragmatic inference. Table 1 presents examples of each of these items. Different kinds of items provided a broad context for the investigation of format effects. Probability judgments with colors and pragmatic inference items seemed, at least at an intuitive level, to depend more on a visual representation than did the other two items. It was of interest to determine whether format effects were characteristic of all items or specific to just a few.

These materials were used to develop a sentence verification task involving true/false reaction times (RTs). The background inputs were presented in either picture or word versions. Figure 1 presents samples of the pictureword inputs. Subjects received one version of the background input on each trial, followed by a test sentence that was either a verbatim trace or an inference. The test sentence was always presented in sentence format, and there was an equal number of true and false instances in which the sentence contained information that was true or false with respect to the input material.

The basic question concerned the format effect. When responding to a verbatim or inference statement, does it matter in which format the subjects receive the background input? Can we reason just as efficiently from words as from pictures? Multimodal models (Potter, 1979; Theios \& Amrhein, 1989b) predict that the original material, once translated into an arbitrary semantic code, would be equally accessible when the test sentence is presented. So, format effects would be minimal. However, according to dual-coding theory (Paivio, 1971, 1978), pictures would have a more privileged access to the semantic network, so a format effect would be predicted particularly when the task requires reasoning. Similarly, a pictorial processing advantage would be predicted from the text-processing literature because pictures facilitate comprehension (Glenberg \& Langston, 1992; Larkin \& Simon, 1987).

Table 1

Sample Stimuli From Each Kind of Item

\begin{tabular}{|c|c|c|c|}
\hline Paradigm & Background Input & Verbatim & Inference \\
\hline $\begin{array}{l}\text { Probability judgment } \\
\text { with colors }\end{array}$ & $\begin{array}{l}7 \text { red squares } \\
5 \text { blue squares } \\
3 \text { yellow squares }\end{array}$ & $\begin{array}{l}\text { True: There are } 7 \text { red squares. } \\
\text { False: There are } 7 \text { blue squares. }\end{array}$ & $\begin{array}{l}\text { Yellow squares are least likely. } \\
\text { Red squares are least likely. }\end{array}$ \\
\hline $\begin{array}{l}\text { Probability judgment } \\
\text { with shapes }\end{array}$ & $\begin{array}{l}3 \text { squares } \\
5 \text { circles } \\
7 \text { triangles }\end{array}$ & $\begin{array}{l}\text { True: There are } 7 \text { triangles. } \\
\text { False: There are } 7 \text { circles. }\end{array}$ & $\begin{array}{l}\text { Triangles are most likely. } \\
\text { Squares are most likely. }\end{array}$ \\
\hline Category inclusion & $\begin{array}{l}3 \text { horses } \\
3 \text { cows } \\
3 \text { roses }\end{array}$ & $\begin{array}{l}\text { True: There are } 3 \text { roses. } \\
\text { False: There are } 3 \text { daisies. }\end{array}$ & $\begin{array}{l}\text { There are fewer flowers than animals. } \\
\text { There are fewer animals than flowers. }\end{array}$ \\
\hline Pragmatic inference & $\begin{array}{l}\text { The circle is } \\
\text { on top of the } \\
\text { star. The arrow } \\
\text { is in the circle. }\end{array}$ & $\begin{array}{l}\text { True: The arrow is in the circle. } \\
\text { False: The star is in the circle. }\end{array}$ & $\begin{array}{l}\text { The star is under the arrow. } \\
\text { The arrow is under the star. }\end{array}$ \\
\hline
\end{tabular}




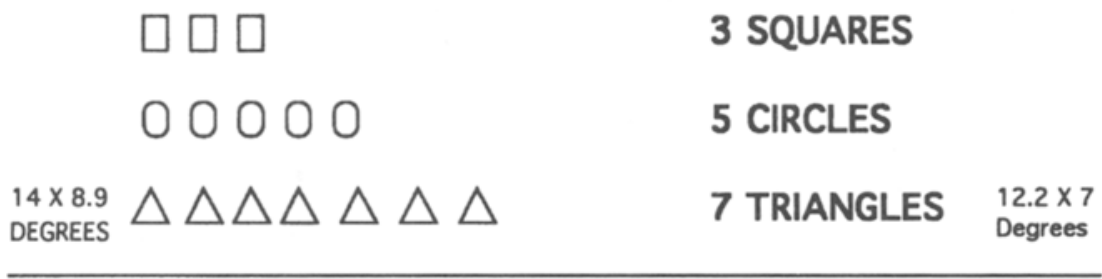

CATEGORY INCLUSION

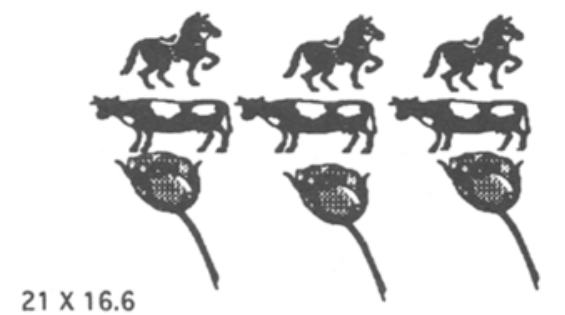

3 HORSES

3 cows

3 ROSES

$12.2 \times 7$

Degrees

PRAGMATIC INFERENCE

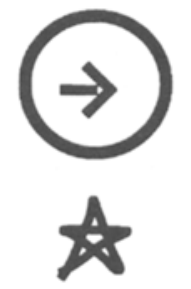

$5.3 \times 8.9$

Degrees
THE CIRCLE IS ON TOP OF

THE STAR.

\section{THE ARROW IS IN THE CIRCLE.}

Figure 1. Samples of the picture and word versions of the background input.

Since the test sentence was always in a word form, there was a difference in compatibility between the picture and word inputs and the test sentence. There was a high degree of compatibility when the word input was presented with a verbatim statement. Verbatim test sentences were identical to parts of the word input except for the addition of the sentence context. In comparison, picture input had a low compatibility. Compatibility was also low with the inference statements from both formats. Generally, differences in stimulus compatibility shortens RT, and, in this study, it would be expected to provide a processing advantage for the word input, particularly with the verbatim test sentences.

\section{EXPERIMENT 1}

In Experiment 1, the background input (either picture or word) was presented for $8 \mathrm{sec}$, followed by a test sentence. Subjects made a true/false RT to the test sentence. Verbatim and inference sentences included both true and false statements for each of the four items identified in
Table 1. Subjects participated in all of the experimental conditions. The analyses tested for effects of format, verbatim/inference item type, test statement accuracy, and kind of problem-solving item, as well as for the interactions of these variables.

The procedure was such that differences in encoding picture and word inputs would not influence RT. The presentation time for the background input was long enough to completely encode the material prior to receiving the test sentence. Differences in RTs would result only from the processing that followed the encoding of the background material.

\section{Method}

Subjects. The subjects were 30 men and women from the University of North Carolina at Charlotte who had normal or correctedto-normal $(20 / 20)$ vision. They participated in the experiment to obtain extra credit points toward their psychology class grade.

Stimulus materials. Picture and word versions of the background input were developed for each kind of problem-solving item. Figure 1 presents some examples. The set of stimuli for the experiment included 4 picture-word inputs for each of the four 
kinds of item. As much as possible, the sizes of the picture-word inputs were equated. In all cases, when viewed from a distance of $30 \mathrm{~cm}$, the stimuli were larger than $3^{\circ}$ of visual angle. The specific dimensions of each of the stimuli are identified in Figure 1. The sizes of the picture and word versions of the probability judgment with color stimuli are approximately the same as the sizes of the stimuli for the probability judgment with shape. In addition to size, Theios and Amrhein (1989a) have criticized picture-word studies for a failure to equate stimuli in visual detail. With complex stimuli, however, it is not entirely clear whether it is possible to equate visual detail without some loss of external validity. Pictures and words are inherently different, and restricting studies to only stimuli that are equated on visual detail, as suggested by Theios and Amrhein (1989b), seriously limits the real-world nature of the stimulus materials. Some of the items (i.e., probability judgment with shapes) have picture-word versions that are closer in visual detail than are others (i.e., category inclusion). Visual detail has been shown to impact stimulus recognition within the first $100 \mathrm{msec}$ after exposure. In Experiment 1 , the background input was presented for $8 \mathrm{sec}$ prior to the presentation of the test sentence. What happened during the first $100 \mathrm{msec}$ was not expected to have a serious impact on the subject's response. The impact of this difference on the RT data from Experiment 2 is discussed in the General Discussion section.

The test sentences were developed from verbatim traces and gists identified by Brainerd and Reyna (1993). The verbatim sentences consisted of material taken directly from the background input. Table 1 presents some true and false examples. For the probability judgment tasks, the sentences tested memory for how many items appeared in a particular shape or color. In the category inclusion example, the verbatim statements tested for memory of a particular category item, and the pragmatic inference item tested memory for which shape was inside another. As indicated previously, there were differences in stimulus compatibility between the test sentences and the picture-word inputs. Compatibility was higher with the word inputs than with the picture inputs.

Inference statements were developed from gists and required some reasoning - that is, the statements were not explicitly presented in the background input. In the probability judgment item, the inferences required the subjects to make judgments of which color or shape was most/least likely. In the category inclusion example, statements questioned whether the background input contained more/fewer examples of members of a particular category. In the pragmatic inference item, the statements required a judgment of whether a particular shape was over/under another when the material in the background input did not state it explicitly. In a sense, the pragmatic inference item was different from the others because the word input required more reasoning than did the picture version. The verbatim and the inference statements were deemed so only with regard to the word version. With the picture version, spatial relationships were quite obviously represented, and there did not seem to be much difference between statements of which shape was inside the other and what was above/below something else. So, in this respect, this item was different from the others. This item was included because of its conceptual similarity to the items tested in the previous work with the sentence-picture verification task (Clark \& Chase, 1972).

The stimuli were displayed on an Apple color high-resolution RGB 13-in. monitor. The monitor has a P22 phosphor with a mediumshort persistence. Stimulus presentation and data collection were controlled by SuperLab running on a Macintosh II computer.

Procedure. Each trial consisted of two stimulus events. The first was the presentation of the background input in either picture or word version for $8 \mathrm{sec}$. Pilot tests showed that $8 \mathrm{sec}$ was sufficient for the subjects to encode either of the versions of the background input. This was followed by a test sentence that remained on the screen until the subject made a keypress response. Both stimulus events appeared in the center of the screen. RTs measured the time period between the presentation of the test sentence and the subject's keypress response.

The subjects, seated $30 \mathrm{~cm}$ from the monitor, participated individually in sessions of approximately $45 \mathrm{~min}$. They used a chinrest to stabilize their head movements. The subjects were instructed to study the material presented on the first screen and to respond to the test sentence as quickly as possible without sacrificing accuracy. The subjects were told to respond "true" if the test sentence contained material that was presented on the first screen or could be inferred from the material on the first screen and to respond "false" otherwise. Responses were made by pressing $\mathrm{T}$ or $\mathrm{F}$ on the keyboard. Each subject participated in six practice trials prior to the experiment.

There were 128 trials that represented 4 replications of 32 experimental conditions. Each subject participated in a random arrangement of trials that represented the 2 formats factorially combined with 2 item types with both true and false statements and 4 kinds of problem-solving items.

\section{Results}

Means and medians were computed from the correct RTs obtained from each subject across the 4 trials within each of the experimental conditions. RTs in excess of $8 \mathrm{sec}$ (less than $2 \%$ of the responses) were not included in the analysis. Also recorded were the proportion of incorrect responses. Data from 2 subjects were excluded because of excessively high error rates. A $2 \times 2 \times 2 \times$ 4 repeated measures analysis of variance (ANOVA) was used on the the RT and error data to test for effects of format, verbatim/inference item type, statement accuracy, and kind of item. The $F$ tests that are reported include the Geisser-Greenhouse correction to protect against possible violation of the homogeneity assumption. Since the analyses of mean and median RTs resulted in the same effects, only the analysis on means is presented.

Input format was found to significantly affect RTs $[F(1,27)=29.16, p=.0001]$; this variable interacted with verbatim/inference item type $[F(1,27)=5.08, p=$ $.03]$ and with item type and statement accuracy $[F(1,27)=$ $5.92, p=.02]$. The nature of these effects can been seen in Figure 2, where the data are presented separately for each of the problem-solving items tested. RTs to the test sentences were longer when background material appeared as words than when background material appeared as pictures, and the format difference was more substantive when inferences were presented rather than verbatim sentences. This format effect was consistently obtained with all 4 kinds of items as indicated by the lack of significance for the four-way interaction $[F(3,81)=2.45$, $p=.08]$.

There was, however, a main effect of kind of item $[F(3,81)=8.42, p=.0001]$; this variable interacted with item type $[F(3,81)=14.23, p=.0001]$, statement accu$\operatorname{racy}[F(3,81)=6.18, p=.0008]$, and in a three-way interaction with item type and statement accuracy $[F(3,81)=$ $3.37, p=.02]$. Although format effects were consistent across the 4 kinds of items, that was not the case with item type differences or statement accuracy differences. From Figure 3, it appears that, for all kinds of items, responding to inferences took longer than did responding 
PROBABILITY JUDGMENT WITH COLORS PROBABILITY JUDGMENT WITH SHAPES

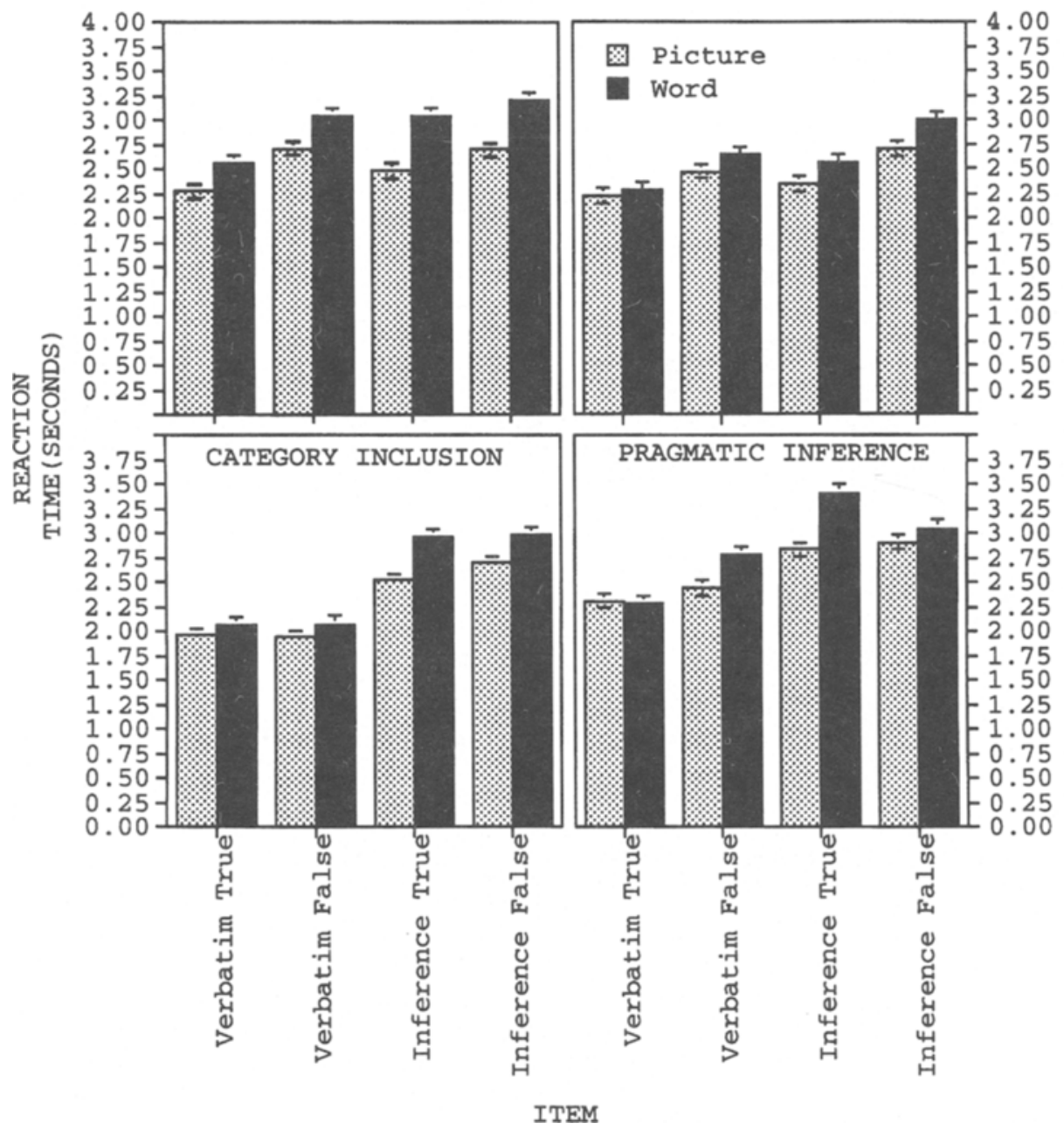

Figure 2. Mean RT as a function of format, item type, statement accuracy, and kind of item in Experiment 1.

to verbatim statements, but the difference between the item types was larger for the category inclusion and the pragmatic inference items than for the two probability judgment items. Moreover, false statements took longer than did true ones, and the true/false difference was larger with the 2 probability judgment items than with the other 2 kinds of items. Although there were 4 kinds of problemsolving items tested, the data show a similar pattern of effects for the 2 probability judgment items and the other 2 items (category inclusion and pragmatic inference). Also, there were overall differences in responding to each kind of item that reflected differences in item difficulty. The pragmatic inference item took the longest, followed closely by probability judgment with color, and probability judgment with shape. The category inclusion item resulted in the quickest RTs. Mean RTs for the 4 kinds of problem-solving items are presented in the order in which they are discussed: $2.772,2.736,2.529$, and $2.409 \mathrm{sec}$.
The RT analysis also showed an item type $\times$ true/false statement interaction $[F(1,27)=4.30, p=.05]$, as well as effects of item type $[F(1,27)=72.64, p=.0001]$ and true/false statement $[F(1,27)=23.13, p=.0001]$. The accuracy of the statement had more of an impact on verbatim sentences than on inference sentences.

Format effects were also apparent from the analysis on errors. There were significant effects of format $[F(1,27)=$ $29.04, p=.0001]$ and item type $[F(1,27)=5.69, p=$ $.02]$, and there was a significant format $\times$ item type interaction $[F(1,27)=17.13, p=.0003]$. From Figure 4, it is apparent that more errors were made to words than to pictures, and the format effect was substantively greater with inference statements than with verbatim statements.

The error analysis also showed an item type $\times$ true/ false statement interaction $[F(1,27)=14.18, p=.0008]$, which is presented in Figure 5 . There were no effects of kind of item $[F(3,81)=1.97, p=.13]$, nor did this variable interact with any of the others. 


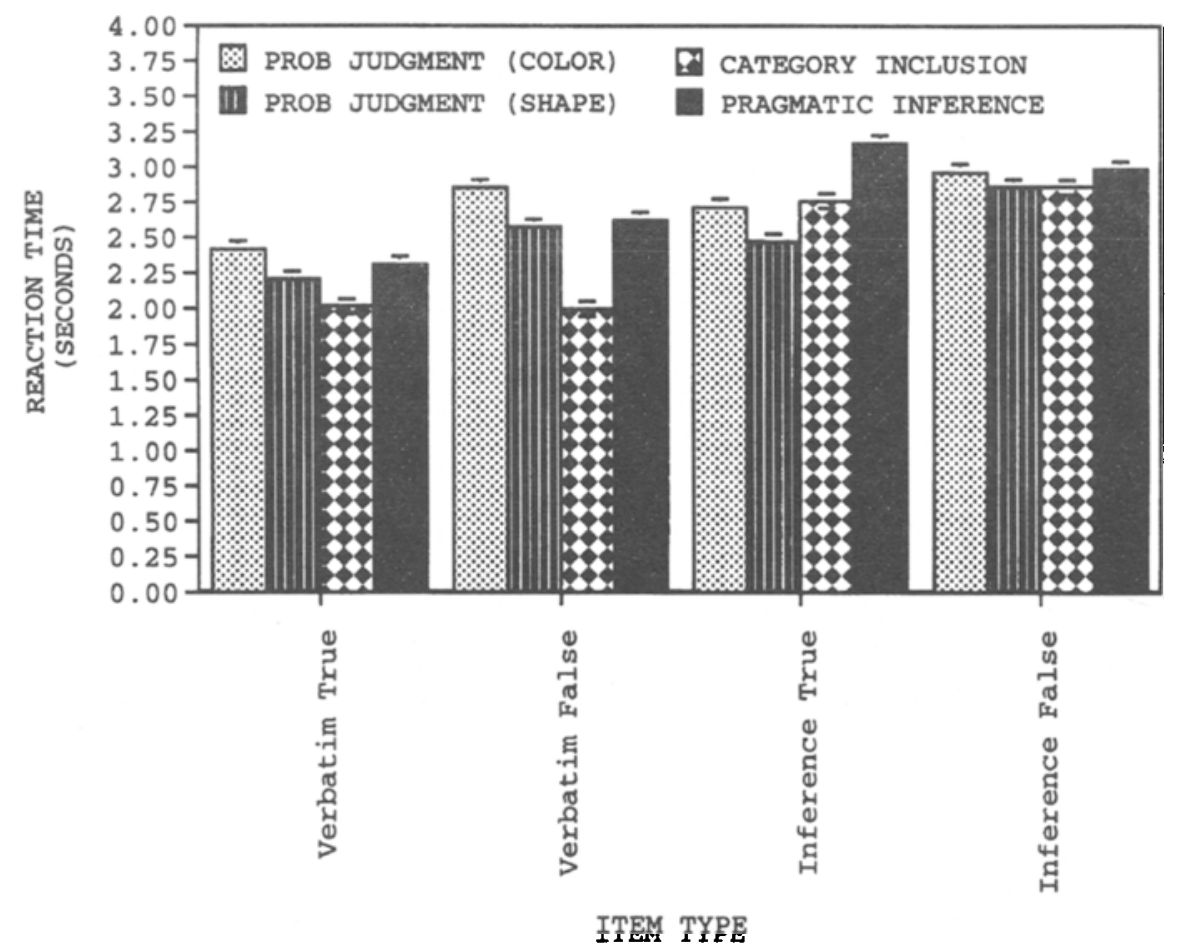

Figure 3. Mean RT as a function of kind of item, item type, and statement accuracy in Experiment 1.

\section{Discussion}

The analyses on both RTs and errors show a consistent advantage when background input appeared as pictures rather than as words. The format effect is also much greater with inferences than with verbatim statements. The finding of a pictorial advantage is consistent with both dualcoding theory (Paivio, 1971) and the literature in text processing (Glenberg \& Langston, 1992; Larkin \& Simon, 1987).

The 8-sec presentation time for the background input may have permitted pictures to be coded in both symbolic and linguistic systems, providing pictures with some advantage relative to word representations. Some difference in access to the semantic network may be suggested by the error data, which showed that when processing words, many more errors were made to inferences than to verbatim statements. However, when processing pictures, there were fewer errors made to inferences than to verbatim statements.

It is also possible that the pictorial advantage resulted from a mental model that provides richer representations than the word input (Glenberg \& Langston, 1992). Or, as Larkin and Simon indicate, the pictorial advantage could result from differences in the way information is extracted.

Interestingly, the format effect was consistent across all of the items studied. Although the items differed in the kind of problem solving that was used, there was a consistent pictorial advantage. The differences that were found among the items were in response to the accuracy of the statement and the item type. The finding of longer RTs to false statements than to true statements is consis- tent with other sentence-picture verification studies (Clark \& Chase, 1972). When compared with true statements, false statements involve an additional stage of processing that lengthens RT. However, test statement accuracy influenced the speed and accuracy of the verbatim judgments more than it influenced the speed and accuracy of the inference judgments. When making decisions based on reasoning rather than memory for recent information, test statement accuracy only minimally influenced the responses. Also, false statements delayed RTs more for the probability judgment items than for the other items, and the difference between the verbatim and inference item types had more of an impact on category inclusion

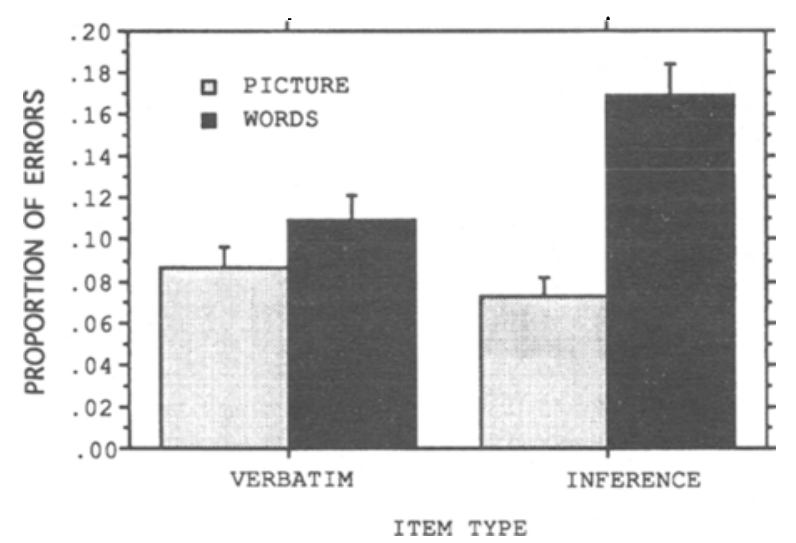

Figure 4. Mean proportion of errors as a function of format and item type in Experiment 1. 


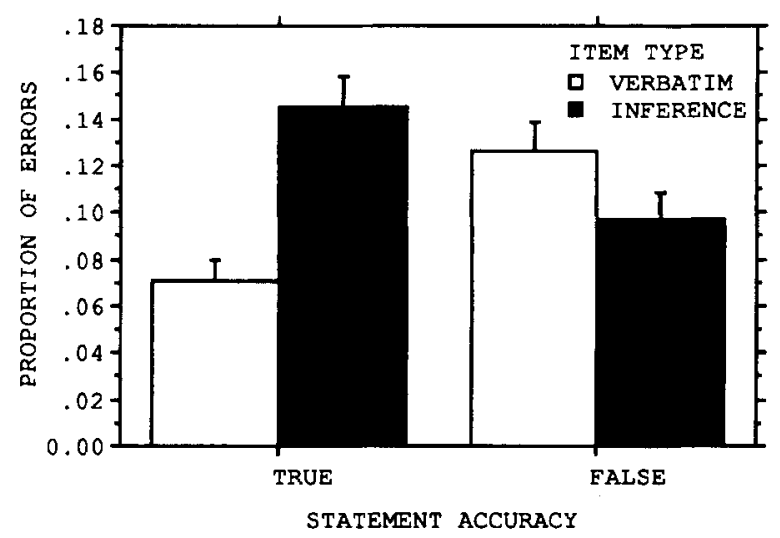

Figure 5. Mean proportion of errors as a function of item type and statement accuracy in Experiment 1.

and pragmatic inference than on the probability judgment items.

As indicated previously, there was some concern, particularly with the pragmatic inference item, that differences between verbatim and inference sentences were not the same when reasoning from picture and word input (i.e., because of the nature of picture representations, verbatim and inference statements may require similar processes). As Larkin and Simon (1987) point out, format differences may result from differences in the explicitness of information between picture and word versions of the same stimulus input. Inferences may require some reasoning in word input but would be directly represented in the picture input. The RT data presented in Figure 2, however, do not support this interpretation. Across all problem-solving items, there was a consistently longer response to inferences than to verbatim sentences with both picture and word inputs. Had inferences been directly represented in the picture input, then there should have been some deviation from that finding on at least one of the items. Since there are none, it is reasonable to suggest that RTs were longer as a result of more processing in response to inference items than to verbatim items. To make sure that the findings represented all of the problem-solving items, the analysis was redone excluding the data from the pragmatic inference item. The format effects were the same except for the absence of the three-way effect of format, item type, and true/ false statement.

The longer RTs to inferences, relative to those to verbatim sentences, are consistent with fuzzy trace theory's assumption (Brainerd \& Reyna, 1992) regarding the qualitative difference in processing between the item types. Inferences take longer because they require reasoning, whereas the verbatim sentences require only that the subject respond to recently encoded information. The finding of stronger format effects with inferences than with verbatim items suggests that pictures facilitate reasoning processes more than they facilitate superficial memory processes. The fact that item type interacted with test statement accuracy and with the 4 kinds of problem- solving items is also consistent with qualitative differences in processing between verbatim and inference responses.

The format effects that were obtained in this experiment differed somewhat from the effects identified by Brainerd and Reyna (1993) when working with children. Brainerd and Reyna found a pictorial advantage for verbatim statements and a word advantage for inference statements, whereas the present findings show a consistent pictorial advantage with both item types. The difference between our results and those of Brainerd and Reyna could be due to task differences. The previous work with fuzzy trace theory used a memory task with accuracy as the primary measure, whereas the present experiment used a sentence verification task with a RT measure. Or the discrepancy in the findings could reflect a developmental difference in processing information. The pictorial advantage in reasoning from inferences may represent a more sophisticated method of processing information that has not been developed in children. More research is needed to clarify this difference in the findings.

Given the nature of the format effect that was obtained, Experiment 2 sought to replicate it and to explain why it was occurring. If the previous results were contingent on sufficient time to encode the background input into a dual format, then the pictorial advantage would be expected only when the background input precedes the test sentence. Subjects use the time prior to the presentation of the test sentence for input processing. However, when the two stimuli appear simultaneously or with a short stimulus onset asynchrony (SOA), then subjects can process the background input in a more selective manner tailored to the information required in the test sentence, and differences in the way that information is extracted from picture and word inputs would be expected to prevail. If a mechanism similar to that found by Larkin and Simon (1987) were occurring with our data, then the findings should show format differences irrespective of when the test sentence is presented, because the format effect would result not from the time course of processing the picture-word input but rather from the way information is extracted from the input.

\section{EXPERIMENT 2}

In Experiment 2, the background input remained on the screen, and the test sentence appeared with varied SOAs from 0 to $1,000 \mathrm{msec}$. It was of interest to test whether the pictorial advantage obtained in Experiment 1 would replicate when the subjects were reasoning from background material that was available on the screen for varying time periods prior to the presentation of the test sentence. So, by manipulating the onset of the test sentence, this experiment investigated whether format effects vary with the time course of processing picture and word inputs.

Picture-word inputs were presented with test statements at SOAs that varied in 200-msec increments from a simultaneous condition up to a delay of $1,000 \mathrm{msec}$. As 
in Experiment 1, the test sentences included both true and false verbatim and inference statements. However, only 2 of the problem-solving items were used: probability judgment with colors and category inclusion. Because the findings from Experiment 1 showed that the 4 kinds of items resulted in two response patterns, it did not appear necessary to test all 4 kinds of items in order to understand the nature of format effects in problemsolving tasks.

\section{Method}

Subjects. The subjects were 10 men and women students from the University of North Carolina at Charlotte. They were volunteers from the author's classes who had normal or corrected-to-normal $(20 / 20)$ vision and no history of visual abnormalities. These subjects were not participants in Experiment 1.

Stimulus materials. The stimulus materials were the same as in Experiment 1; however, only the probability judgment with color and category inclusion items were used.

Procedure. As in Experiment 1, there were two stimulus events. However, the events were presented with the following SOAs: 0, $200,400,600,800$, and $1,000 \mathrm{msec}$. To control precisely the time to encode the picture and word versions of the background input, a 600 -msec wait was used before presenting the first stimulus event (the background input). This action was necessary because it took longer for the drawing of the picture than for the word input to the screen. That is, in the category inclusion example, about $400 \mathrm{msec}$ were needed to draw the picture, whereas $50 \mathrm{msec}$ were needed for the word version. In effect, the inclusion of the wait at the beginning of each trial kept the screen invisible until the image was drawn and ready to be seen. In Experiment 1, this was unnecessary because the subjects were given 8 sec to view the background input.

The screen locations of the two stimulus events were also adjusted so that both could be viewed simultaneously. Instead of each appearing in the center of the screen (as in Experiment 1), the background input appeared in the upper center portion of the screen and the test sentence appeared in the lower center. RTs measured the time period between the presentation of the test sentence and the subject's keypress.

There were 384 trials, representing 4 replications of 96 experimental conditions. It took about 50 min to participate in the experiment. Each subject participated in a random arrangement of the trials that represented the 2 formats factorially combined with 2 item types, false and true statements, 2 kinds of items, and 6 SOAs.

\section{Results}

Means were computed from the correct RTs obtained from each subject across the 4 trials within each of the experimental conditions. RTs in excess of $8 \mathrm{sec}$ (less than $2 \%$ of the responses) were not included in the analysis. Also recorded were the proportion of incorrect responses. A $6 \times 2 \times 2 \times 2 \times 2$ repeated measures ANOVA was used to test for the effects of SOA, format, verbatim/inference item type, statement accuracy, and kind of problemsolving item. The $F$ tests include the Geisser-Greenhouse correction to protect against violation of the homogeneity assumption.

As expected, RTs were found to decrease with increasing SOA $[F(5,45)=8.92, p=.0001]$. SOA was found to interact with accuracy of the test statement $[F(5,45)=2.76, p=.03]$, with format and accuracy of the test statement $[F(5,45)=2.65, p=.0001]$, with format and kind of item $[F(5,45)=2.63, p=.04]$, and in a four-way effect with format, accuracy, and kind of item $[F(5,45)=3.44, p=.01]$. The four-way interaction is presented in the two panels of Figure 6 . The top panel presents the data for the probability judgment with color item, and RTs to the category inclusion item are presented in the bottom panel.

The format and the kind of item effects were consistent with the results of Experiment 1. Responses to pictures were quicker than to words $[F(1,9)=72.37, p=$ $.0001]$. Format was found to interact with item type $[F(1,9)=54.48, p=.0001]$, with accuracy of the test statement $[F(1,9)=8.28, p=.02]$, and with item type and kind of item $[F(1,9)=21.91, p=.001]$. As can be seen in Figure 7, the format effect was larger with inference statements than verbatim statements; this effect did vary with kind of item. Probability judgments with color were slightly faster than category inclusion responses $[F(1,9)=9.36, p=.013]$. Kind of item interacted with item type $[F(1,9)=65.99, p=.0001]$ and in a three-way interaction with item type and accuracy of test statement

\section{PROBABILITY JUDGMENT WITH COLORS}

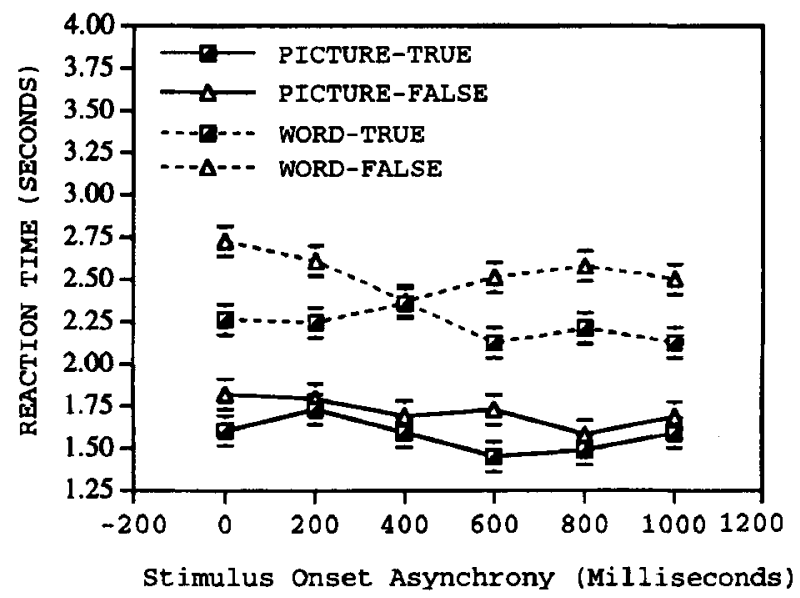

CATEGORY INCLUSION

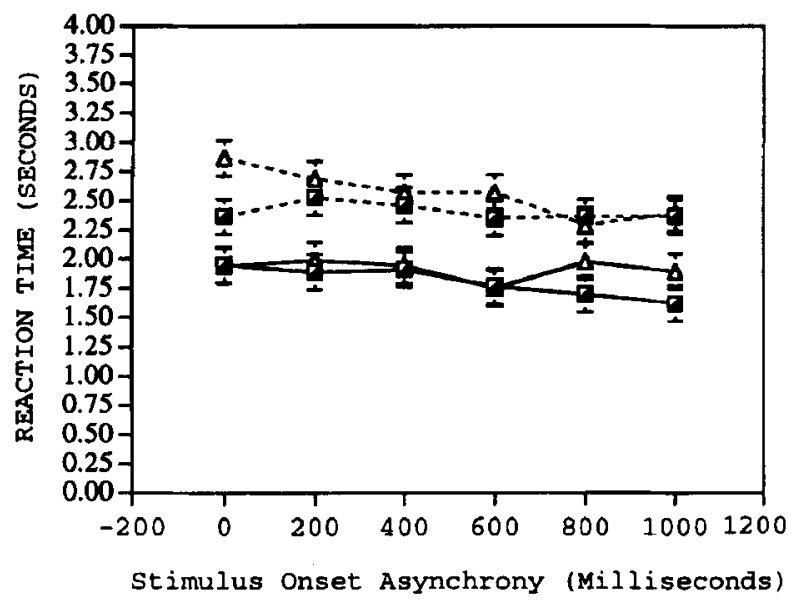

Figure 6. Mean RT as a function of format, statement accuracy, kind of item, and SOA in Experiment 2. 
$[F(1,9)=8.88, p=.01]$. Consistent with the findings of Experiment 1, probability judgments showed a smaller verbatim/inference item type difference than did category inclusion judgments.

Mean RTs from comparable conditions of Experiment 1 (Figure 2) and Experiment 2 (Figure 7) show faster responses (about $500 \mathrm{msec}$ ) to test sentences when the background input is visible for all conditions except when making inferences from word inputs regarding category inclusion. In this condition, RTs were the same in the two experiments.

Analysis of the errors did not show the format effect obtained in Experiment $1[F(1,9)=1.55, p=.24]$. However, format was found to interact with item type $[F(1,9)=$ $15.40, p=.003]$, item type and kind of item $[F(1,9)=$ $6.30, p=.03]$, and item type, kind of item, and SOA $[F(5,45)=3.45, p=.01]$. The four-way interaction is presented in Figure 8. The upper panel reports the proportion of errors made in response to the probability judgment items, and the lower panel reports the error rate for the category inclusion items. For most conditions, with the exception of word inferences with the category inclusion item, the error rate was smaller than the error rate obtained in Experiment 1. It is not surprising that performance would be more accurate in Experiment 2 because the background input remained on the screen as the subject responded to the test sentence. In Experiment 1, the subjects needed to depend upon their memory representations for comparisons with the test sentences.

\section{Discussion}

The findings from Experiment 2 show consistent format difference across SOA conditions. RTs to pictures were faster than those to words even when test sentences appeared simultaneously with the background input. This finding occurred even though there were differ-

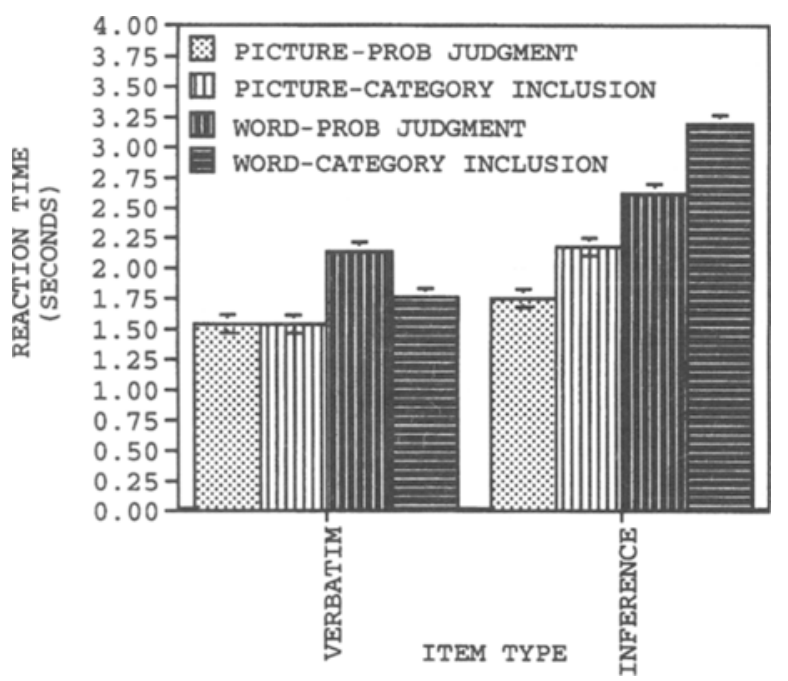

Figure 7. Mean RT as a function of format, item type, and kind of item in Experiment 2.
PROBABILITY JUDGMENT WITH COLORS

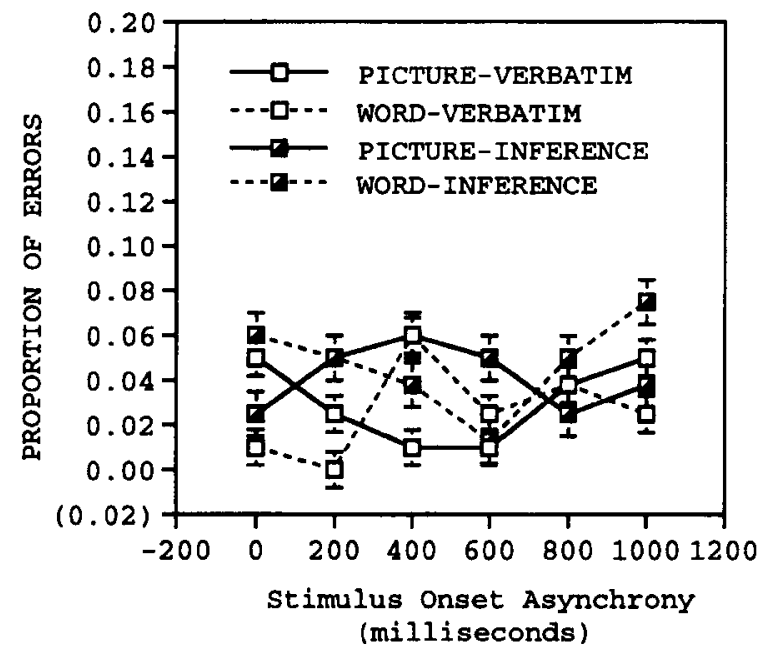

CATEGORY INCLUSION

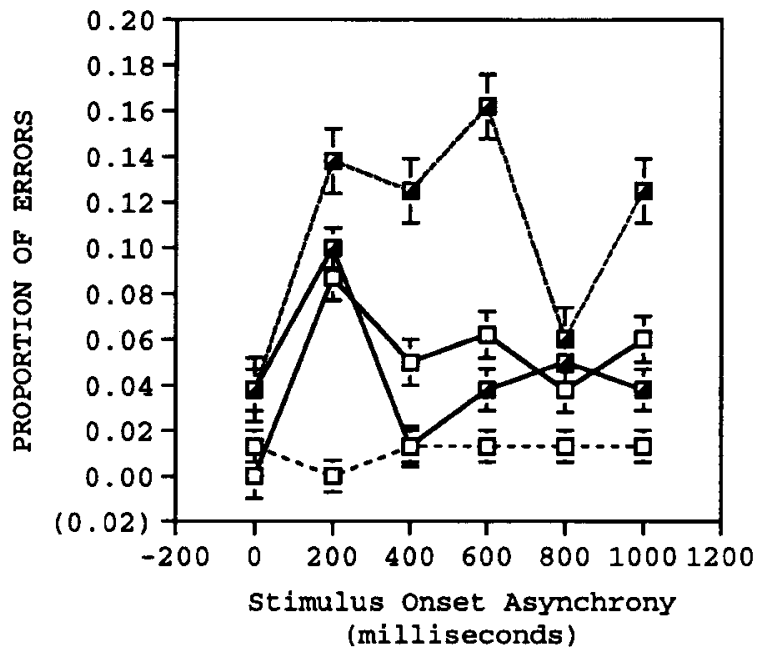

Figure 8. Mean proportion of errors as a function of format, item type, kind of item, and SOA in Experiment 2.

ences in compatibility between the test sentence and the picture and word input. The high degree of compatibility between the verbatim sentence and the word input did not result in faster RTs relative to the picture input.

The locus of the format effect appears to reside in the fact that information is more readily accessible from pictures than from words, and the difference is more evident when reasoning is required, relative to verbatim responses. Somehow, pictures provide a more efficient representation than do words.

When compared with the subjects in Experiment 1, the subjects in Experiment 2 were quicker and more accurate in their responses to all conditions except one: when making inferences from category inclusion items with word inputs. The better performance resulted from the fact that the background input was always present on the screen. Responding to test sentences when the input 
was readily available shortened RTs by about $500 \mathrm{msec}$, relative to comparable conditions in which test sentences were compared with representations of inputs stored in memory.

In general, the data from Experiment 2 replicated the findings from Experiment 1 and suggest that the pictorial advantage occurs whether the test sentence appears together with the background input or is presented with up to a $1,000-\mathrm{msec}$ delay. The pictorial advantage is obtained whether reasoning from memory as in Experiment 1 or from material that is available on the screen at the time that the test sentence appears. Implications of these results are discussed in the next section.

\section{GENERAL DISCUSSION}

The data from Experiment 2 replicated the findings from Experiment 1 and show that the picture advantage does not vary with the SOA of the test sentence. The picture advantage seems to be due to the nature of the input and how we extract information from it rather than access to semantic memory as suggested by dual-coding theory. There are several text-processing theories that are consistent with these data. For example, Larkin and Simon (1987) theorize that text and diagrams containing the same information are not necessarily equivalent in terms of the processing required to extract the information. The operations working on one representation may recognize features readily or make inferences directly that are difficult in the other representation. This interpretation is consistent with the data from Experiments 1 and 2. The pictorial advantage is obtained whether reasoning from memory or from material that is available on the screen at the time that the test sentence appears.

These data are also consistent with Glenberg and Langston's (1992) suggestion that pictures help the comprehension and retention of text through working memory management. Pictures assist in the construction and management of a representation that is richer and more elaborate than would ordinarily be available from text. Viewing a picture may provide a relatively effortless maintenance of some of the representational elements corresponding to parts in the picture, freeing up capacity for inference generation. This may explain why our results show that people are faster and more accurate at making an inference when reasoning from pictures than when reasoning from words. It appears that the format effect originally obtained with simple stimuli (i.e., category names and color-word stimuli) can be generalized to a more complex task involving problem solving.

Interestingly, the format effects are the same whether the subjects are reasoning from background input that is available on the screen or reasoning from a representation of that input. Although this manipulation affected the speed and accuracy of the overall task response, it did not affect the pattern of the findings, particularly with respect to format effects. Picture inputs are more efficient, at least in the sense that they facilitate the reasoning that is needed for probability judgments with colors and shapes, for category inclusion, and for pragmatic inference items.

Although the findings from Experiment 1 suggest that the format effect may be because pictures are coded in both verbal and nonverbal representations (as suggested by Paivio), this is an unlikely explanation for the results of Experiment 2, since reasoning occurred when the original materials were readily available rather than through a coded representation. Moreover, when both input and test sentence appeared simultaneously, there would not have been sufficient time to dual code the picture inputs.

These findings do not support the notion that format effects should occur only in certain tasks, such as naming or drawing or when there is a difference in the size or visual detail of the picture-word versions of the stimuli (Theios \& Amrhein, 1989a, 1989b). If the pictorial advantage was due to better recognition of the picture input during the first $100 \mathrm{msec}$ of processing, then, in Experiment 2, the format effect would have been limited to the conditions in which the test sentence appeared simultaneously with or shortly after the presentation of the input. How could encoding differences explain the format effect in Experiment 1 when subjects had a full $8 \mathrm{sec}$ to study the background input prior to the test sentence, or even in Experiment 2 when background input was available for a full second prior to the test sentence. Also, such an explanation would have required some inconsistency in the format effect across items because the pictureword inputs for items such as probability judgment with shape were much closer in visual detail than were others. The findings consistently show a performance advantage when pictures appeared, relative to when words appeared, under varied presentation conditions and with varied problem-solving items.

The 4 kinds of items showed similar format effects but varied in response to the accuracy of the statement and effect of item type. Probability judgments with either shape or color were influenced by the accuracy of the test statement. False statements delayed RTs. The finding of a longer RT with false statements, as compared with true statements, was consistent with findings of Clark and Chase (1972). False statements, relative to true statements, delay RTs because of an additional processing stage. The true/ false difference, however, was more evident with the probability judgment items than with the other kinds of items tested in this study. It is important to note that only in this regard were the results of this study similar to Clark and Chase (1972). The model of sentence-picture verification developed from their data suggest that both sentences and pictures were matched through a series of discrete stages involving abstract and amodal representations. The findings of the present experiments suggest a pictorial processing advantage. However, as indicated in the introduction, the procedure used in these experiments extended the procedure developed by Clark and Chase in many important ways, and it is not surprising that the findings differed.

The 4 kinds of problem-solving items also differed in responses to verbatim and inference sentences. When com- 
pared with the probability judgment items, problem solving with category inclusion and pragmatic inference items seemed more affected by whether the test statement was a verbatim or an inference sentence. The fact that inferences took longer than verbatim judgments supports the contention of fuzzy trace theory that responding to gist statements is qualitatively different from responding to verbatim statements. Inferences take longer because they are endogenous and involve reasoning, whereas verbatim statements are exogenous and arise from recently encoded information. Also, the fact that verbatim and inference differences were found when the subjects were reasoning from both picture and word inputs refutes the argument that the distinction between the item types was more characteristic of the word inputs than the picture inputs. The findings from Experiment 2 are consistent with those of Experiment 1 in showing similar verbatim/inference differences to picture and word inputs.

In conclusion, the findings of these experiments show that people are faster at drawing inferences from pictures than from words. This format effect is also present with verbatim judgments, but the picture-word difference is not as substantial.

\section{REFERENCES}

BRAINERD, C. J., \& REYNA, V. F. (1992). Explaining "memory free" reasoning. Psychological Science, 3, 332-339.

Brainerd, C. J., \& ReYNA, V. F. (1993). Memory independence and memory interference in cognitive development. Psychological Review, $100,42-67$.

CARPenter, P. A., \& Just, M. A. (1975). Sentence comprehension: A psycholinguistic processing model of verification. Psychological Review, 82, 45-83.

Clark, H. H., \& Chase, W. G. (1972). On the process of comparing sentences against pictures. Cognitive Psychology, 3, 472-517.

Glaser, W. R., \& Glaser, M. O. (1989). Context effects in Stroop-like word and picture processing. Journal of Experimental Psychology: General, 118, 13-42.
Glenberg, A. M., \& Langston, W. E. (1992). Comprehension of illustrated text: Pictures help to build mental models. Journal of Memory \& Language, 31, 129-151.

Goolkasian, P., \& Park, D. C. (1980). Processing of visually presented clock times. Journal of Experimental Psychology: Human Perception \& Performance, 6, 707-717.

Kroll, J. F., \& CORRIGAN, A. (1981). Strategies in sentence-picture verification: The effect of an unexpected picture. Journal of Verbal Learning \& Verbal Behavior, 20, 515-531.

LARKIN, J. H., \& SimON, H. A. (1987). Why a diagram is (sometimes) worth ten thousand words. Cognitive Science, 11, 65-99.

PAIvio, A. (1971). Imagery and verbal processes. New York: Holt, Rinehart \& Winston.

Paivio, A. (1975). Perceptual comparisons through the mind's eye. Memory \& Cognition, 3, 635-647.

PAIvio, A. (1978). A dual coding approach to perception and cognition. In H. L. Pick \& E. Saltzman (Eds.), Modes of perceiving and processing information (pp. 39-51). Hillsdale, NJ: Erlbaum.

Pellegrino, J. W., Rosinski, R. R., Chiesi, H. L., \& Siegel, A. (1977) Picture-word differences in decision latency: An analysis of single and dual memory models. Memory \& Cognition, 5, 383-396.

POTTER, M. C. (1979). Mundane symbolism: The relations among objects, names, and ideas. In N. R. Smith \& M. B. Franklin (Eds.), Symbolic fuctioning in childhood (pp. 41-65). Hillsdale, NJ: Erlbaum.

PotTer, M. C., \& Faulconer, B. A. (1975). Time to understand pictures and words. Nature, $\mathbf{2 5 3}, 437-438$.

SEYMour, P. H. (1973). A model for reading, naming, and comparison. British Journal of Psychology, 64, 35-49.

SMith, M. C., \& MAGEE, L. E. (1980). Tracing the time course of picture-word processing. Journal of Experimental Psychology: General, 109, 373-392.

SNODGRASS, F. G. (1984). Concepts and their surface representations. Journal of Verbal Learning \& Verbal Behavior, 23, 3-22.

Theios, J., \& AMrhein, P. C. (1989a). The role of spatial frequency and visual detail in the recognition of patterns and words. In C. Izawa (Ed.), Current issues in cognitive processes (pp. 389-409). Hillsdale, $\mathrm{NJ}$ : Erlbaum.

Theios, J., \& Amrhein, P. C. (1989b). Theoretical analysis of the cognitive processing of lexical and pictorial stimuli: Reading, naming, and visual and conceptual comparisons. Psychological Review, 96, 5-24.

(Manuscript received December 2, 1994; revision accepted for publication August 10, 1995.) 